\title{
Survivin withdrawal by nuclear export failure as a physiological switch to commit cells to apoptosis
}

\author{
K-S Chan ${ }^{1,3}$, C-H Wong ${ }^{1,3}$, Y-F Huang ${ }^{1,2}$ and H-Y Li ${ }^{\star, 1}$
}

Apoptosis is a tightly controlled process regulated by many signaling pathways; however, the mechanisms and cellular events that decide whether a cell lives or dies remain poorly understood. Here we showed that when a cell is under apoptotic stress, the prosurvival protein Survivin redistributes from the cytoplasm to the nucleus, thus acting as a physiological switch to commit the cell to apoptosis. The nuclear relocalization of Survivin is a result of inefficient assembly of functional RanGTP-CRM1-Survivin export complex due to apoptotic RanGTP gradient collapse. Subsequently, Survivin undergoes ubiquitination, which not only physically prevents its diffusion back to the cytoplasm but also facilitates its degradation. Together, this spatial and functional regulation of Survivin abolishes its cytoprotective effect toward the apoptotic executors and thus commits a cell to apoptosis. Our data indicate that the withdrawal of Survivin is a novel and active physiological regulatory mechanism that tilts the survival balance and promotes the progression of apoptosis.

Cell Death and Disease (2010) 1, e57; doi:10.1038/cddis.2010.34; published online 22 July 2010

Subject Category: Immunity

The significance of apoptosis is never undermined given its central role in normal development and homeostasis of multicellular organism. Apoptosis progression relies on the interplay between prosurvival factors and proapoptotic factors, which crucially decides whether a cell under stress lives or dies. The family of evolutionary conserved prosurvival proteins, inhibitor of apoptosis proteins (IAPs), is essential in protecting cells from entering apoptosis by negative regulation of downstream caspases. ${ }^{1-5}$ Survivin $(16.5 \mathrm{kDa})$ is the smallest member of the IAP family containing a single baculovirus IAP repeat motif and it is frequently overexpressed and/or deregulated in most neoplastic tissues. ${ }^{6-10}$ This makes Survivin a leading research candidate in the development of prognostical tool and a prime target in cancer therapy. ${ }^{11,12}$

As a member of the IAP, Survivin exhibits antiapoptotic characteristics. Elevated level of Survivin has been reported to confer radiation resistance, whereas artificial knockdown of Survivin sensitizes and renders cells more susceptible to irradiation-induced cell death. ${ }^{13,14}$ Survivin is able to inhibit the apoptotic executors; for example, caspases, due to its cytoplasmic localization. It is actively and rapidly exported into the cytoplasm, as Survivin contains a canonical nuclear export signal (NES) domain. The exclusion of Survivin from the nucleus is mediated by the classic nuclear export mechanism involving the interaction between the transport receptor chromosomal region maintenance-1 (CRM1) and Ran-guanosine $5^{\prime}$-triphosphate (RanGTP). ${ }^{15-17}$ On a different note, forced entry of modified Survivin mutants into the nucleus by different groups has been associated to ubiquitination-mediated proteolysis by $26 \mathrm{~S}$ proteasome ${ }^{18-20}$

Theoretically, the presence of prosurvival factors in the cell renders the cell resistant to apoptotic stimuli. Hence, it is conceivable that modulatory mechanisms are in place to negate the effect of the prosurvival proteins and to intensify the activation of proapoptotic proteins for the progression of apoptosis. However, the fate and functionalities of existing prosurvival factors and proteins are incompletely elucidated during cell death. The study herein describes a novel regulatory pathway serving as a physiological switch to mediate the removal of prevailing prosurvival protein Survivin during the onset of apoptosis by the impairment of nuclear export processes that actively contributes toward the eventual demise of the cell.

\section{Results}

Prosurvival protein Survivin is degraded during the initial stages of apoptosis. We believed that in dying cells, the tilt in balance and the convergence of the proapoptotic factors could be due to the removal and/or inactivation of the prosurvival factors. Thus, we sought to characterize prosurvival proteins that might be affected in apoptotic cells. Survivin is a suitable candidate not only because it is highly expressed in cancer cells but also as an IAP, it is also a vital

\footnotetext{
'Division of Molecular and Cell Biology, School of Biological Sciences, College of Science, Nanyang Technological University, Singapore 637551, Singapore *Corresponding author: H-Y Li, Division of Molecular and Cell Biology, School of Biological Sciences, College of Science, Nanyang Technological University, Singapore 637551, Singapore. Tel: + 656316 2931; Fax: + 656791 3856; E-mail: hyli@ntu.edu.sg

${ }^{2}$ Current address: Temasek Laboratories, 5A, Engineering Drive 1, No. 09-02, National University of Singapore, Singapore 11741, Singapore

${ }^{3}$ These authors contributed equally to this work.

Keywords: apoptosis; Survivin; RanGTP; nuclear transport; ubiquitination

Abbreviations: CFP, cyan fluorescence protein; CHX, cyclohexamide; CRM1, chromosomal region maintenance-1; FRET, fluorescence resonance energy transfer; GFP, green fluorescent protein; RanGTP, Ran-guanosin 5'-triphosphate; IAP, inhibitor of apoptosis protein; MST1, mammalian STE20-like protein kinase 1; NES, nuclear export signal; NLS, nuclear localization signal; NPC, nuclear pore complex; XIAP, X-linked Inhibitor of apoptosis protein; YFP, yellow fluorescence protein Received 10.2.10; revised 26.5.10; accepted 10.6.10; Edited by RA Knight
} 
modulator of apoptosis. 5,15,21 Western blot revealed a marked decrease of total endogenous Survivin level in a time-dependent manner (Figure 1a and Supplementary Figure S1a) upon the induction of apoptosis (Figure $1 \mathrm{~b}$ and Supplementary Figure S1b). It is possible that the decline of endogenous Survivin could be due to the decrease of Survivin mRNA transcripts, or the post-translational degradation of the protein or both. To address the likelihood of Survivin being regulated at the transcription level during apoptosis, we performed semiquantitative reverse transcription-PCR. Using glyceraldehyde-3-phoshate dehydrogenase (GAPDH) as an internal control, the level of Survivin mRNA appeared to be relatively stable and unaffected during apoptosis (Figure 1c). Hence, this effectively rules out apoptotic downregulation of Survivin mRNA transcription.

Survivin turnover has been shown to be modulated by the ubiquitin-proteasome proteolytic pathway. ${ }^{20}$ To investigate whether Survivin is similarly subjected to ubiquitin-related degradation upon apoptosis induction, we performed Survivin stability assay as denoted by the schematics in Figure 1d. MG132 is used to block the proteosomal activity and cyclohexamide $(\mathrm{CHX})$ inhibits the synthesis of new proteins. We found that endogenous Survivin proteins are degraded at a faster rate in dying cells as compared with control cells following the resumption of proteasome activity (Figure 1e). Moreover, graphical representation (Figure 1f) clearly showed the prominent decrease in the stability of endogenous Survivin during apoptosis.

Survivin redistributes into the nucleus of early apoptotic cells due to the collapse of RanGTP gradient. As Survivin undergoes ubiquitin-dependent proteolysis in the nucleus, ${ }^{19}$ it is possible that Survivin relocation occurs during the progression of apoptosis. To test out this idea, immunofluorescence staining was performed against Survivin and mAb414, marker for nuclear pore protein complexes (NPCs). Indeed, we found that endogenous Survivin redistributes from the cytoplasm into the nucleus of apoptotic cells in a

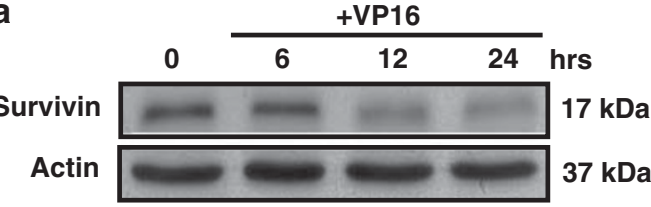

Total Lysates

b

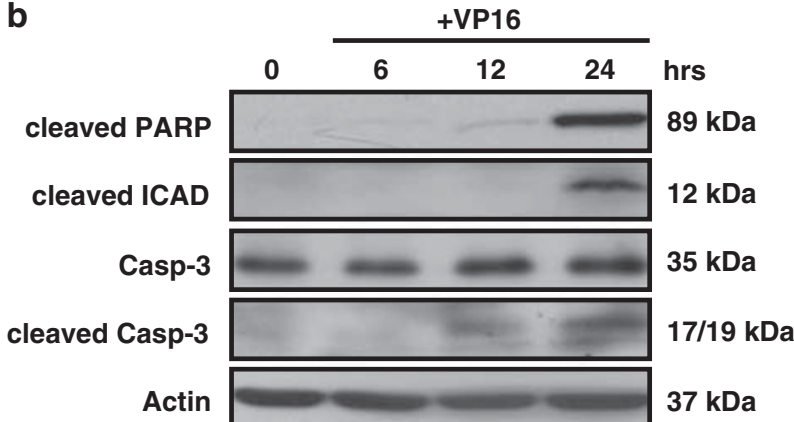

C

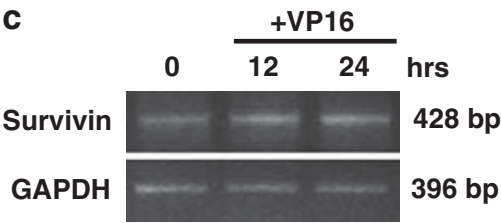

d

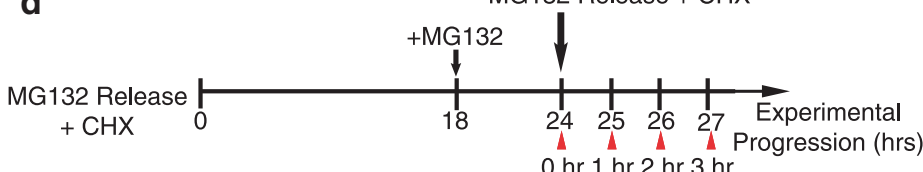
$0 \mathrm{hr} 1 \mathrm{hr} 2 \mathrm{hr} 3 \mathrm{hr}$

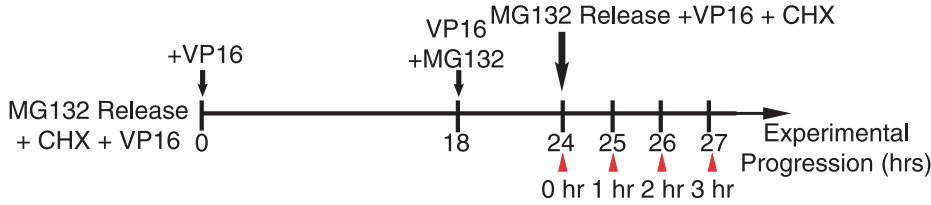

e
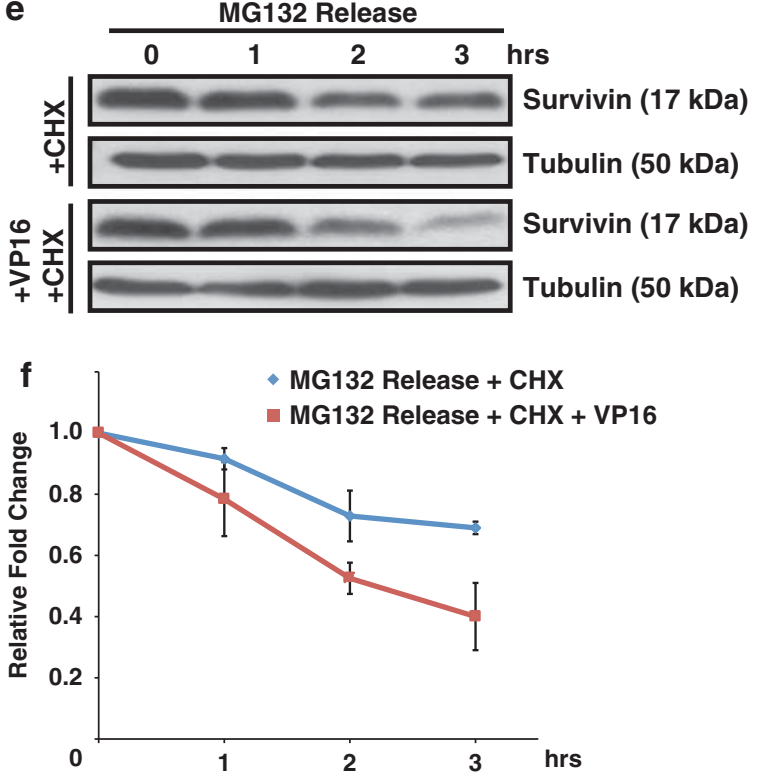

Figure 1 Apoptotic degradation of prosurvival protein Survivin (a) Total cell lysates collected at the stated time points after VP16 treatment and immunoblotted with antiSurvivin and anti-actin (loading control). (b) VP16 treatment-induced cell death as shown by immunoblots of cleaved Caspase-3, cleaved PARP and cleaved ICAD. (c) Total RNAs isolated from HeLa cells treated with or without VP16 at the indicated time points. Semiquantitative reverse transcription-PCR analysis was then performed using GAPDH as internal control. (d) Schematic representation of Survivin stability assay. (e) Experiments performed as shown by the schematics in (d). Endogenous Survivin level was probed at indicated time points after the resumption of the proteosome activity. Actin as loading control. (f) Quantified graphical data presented as mean \pm S.D. (error bar) of three independent experiments 
a

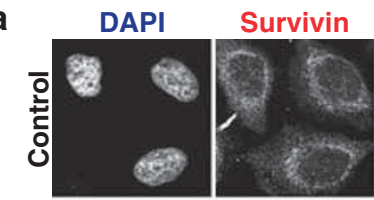

DAP
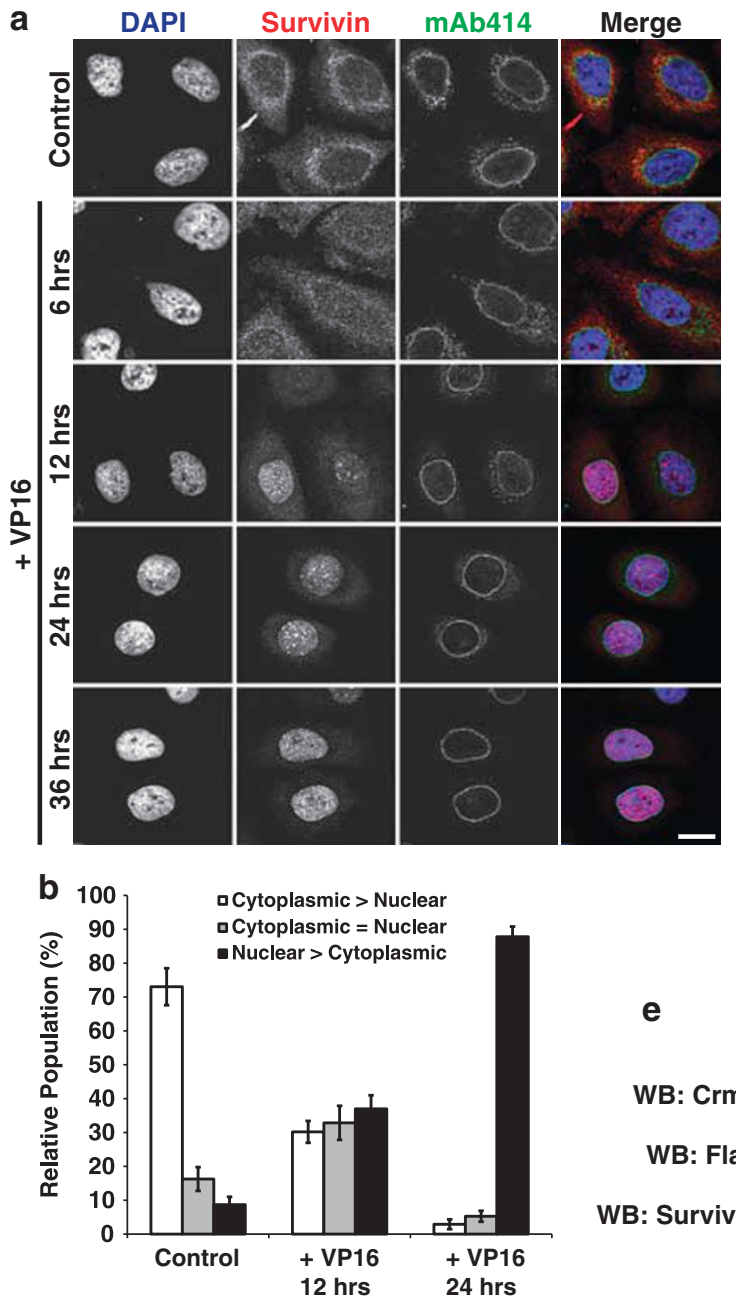
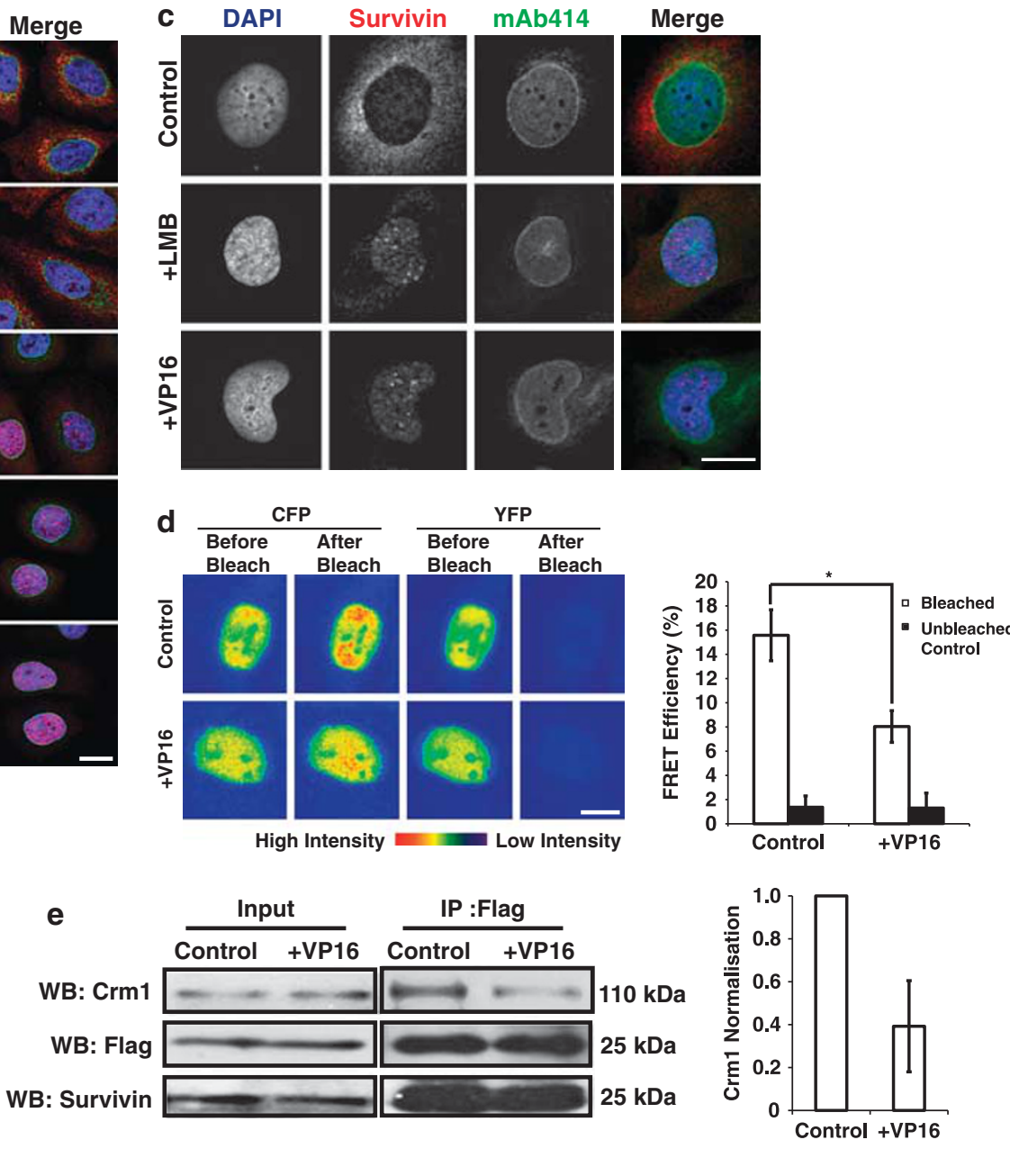

Figure 2 RanGTP gradient collapse induces Survivin redistribution into the nucleus during apoptosis. (a) Survivin and mAb414 staining after VP16 treatment at indicated time points. Images acquired with auto-optimal exposure mode. (b) Quantification of cells with distinctive Survivin localization across the nucleocytoplasmic compartments. Results are presented as mean population percentage of HeLa cells having distinctive Survivin localization \pm S.D. (error bar) of three independent experiments $(n>300$ cells). (c) Immunostaining of non-treated, and treated HeLa cells with LMB or VP16 against Survivin and mAb414. (d) FRET analysis of the nuclear RanGTP gradient by Rango biosensor (fusion protein of CFP and YFP flanking an importin $\beta$ binding domain). Rango undergoes FRET in the presence of RanGTP. Representative images (left) and quantified data (right) presented as mean \pm S.D. (error bar) of three independent experiments $(n=120)$. ${ }^{*} P<0.001$ (Student's $t$-test). (e) Flag-Survivin was overexpressed, immunoprecipitated with anti-Flag from cells incubated with or without VP16 for $24 \mathrm{~h}$. Bars, $10 \mu \mathrm{m}$. (right) Quantified Crm1 intensities normalized against Flag was presented as relative fold change \pm S.D. (error bar) of three independent experiments

a time-dependent manner (Figure 2a and Supplementary Figure S2a). The nuclear envelope appeared to be intact throughout the course of experiment, thus indicating that its function as a physical barrier is not compromised. NPC has been reported to be the substrates of caspases only during late apoptosis. ${ }^{22,23}$ To demonstrate that the relocation of Survivin is a widespread phenomenon, we quantitate the relative percentage of populations of HeLa cells having distinctive Survivin localization. Consistently, control HeLa cells showed predominantly cytoplasmic (cytoplasmic > nuclear) localization of Survivin, ${ }^{4,24}$ whereas the induction of apoptosis triggered a significant increase (Student's $t$-test, $P<0.001)$ in the population showing predominantly nuclear (nuclear $>$ cytoplasmic) localization of Survivin (Figure $2 \mathrm{~b}$ and Supplementary Figure S2b). This suggests that endogenous Survivin redistributes into the nucleus during the early stages of apoptosis.

The nucleocytoplasmic shuttling ability of Survivin is coordinated by passive diffusion and also the presence of an intrinsic NES motif that directs Survivin export into the cytoplasm by CRM1 ${ }^{15,16,18,21}$ Hence, normal circumstantial diffusion of Survivin into the nucleus would not lead to its degradation as it is actively and rapidly exported back into the cytoplasm. Immunostaining of Survivin and mAb414 performed on Leptomycin B (LMB) (potent CRM1 inhibitor) or VP16-treated HeLa cells showed analogous nuclear localization of endogenous Survivin as compared with control (Figure 2c), hinting a possible error along RanGTP-CRM1Survivin axis. Given the importance of the RanGTP gradient in nucleocytoplasmic trafficking, we hypothesized that the 
nuclear export pathway could similarly be affected when the RanGTP gradient collapsed upon apoptosis induction. Using the acceptor photobleaching method of fluorescence resonance energy transfer (FRET), the nuclear RanGTP level was measured as the relative percentage of FRET efficiency. We showed that apoptosis induction results in a significant reduction of nuclear RanGTP level in HeLa cells (Figure 2d and Supplementary Figure S3).

To investigate the interaction between Survivin and CRM1 during apoptosis, Flag-tagged Survivin was overexpressed and immunoprecipitated using anti-Flag. Our data revealed that the interaction between CRM1 and Flag-Survivin was substantially weaker in the presence of apoptotic stimuli (Figure 2e). This indicates that the apoptotic accumulation of nuclear Survivin is driven by nuclear export deregulation by the RanGTP-CRM1 pathway.

Re-establishment of RanGTP gradient rescues Survivin redistribution. We speculated the possibility that the export of endogenous Survivin into the cytoplasm could resume if the RanGTP gradient recovers. In our earlier report, we have established the use of mammalian STE20-like protein kinase 1 (Mst1) siRNA to restore diminished nuclear RanGTP level. ${ }^{25}$ Hence, by using the same strategy, we sought to find out whether Survivin could be exported to the cytoplasm in the presence of apoptotic stimuli after Mst1 knockdown. Western blot was performed on mock-transfected cells and cells transfected with Mst1 siRNA or control siRNA to verify the depletion of MST1 protein level. Treatment of Mst1 siRNA achieved $~ 80 \%$ knockdown efficiency, but does not affect Survivin and Ran (Figure 3a). Consistently, the depletion of MST1 restores nuclear RanGTP in cells treated with VP16 (Figure 3b). A clear indication of RanGTP gradient collapse is the mislocalization of Ran from predominantly nuclear to being dispersed across the nucleocytoplasmic compartment. As shown in Supplementary Figure S4a and b, Mst1 knockdown restores the nuclear localization of Ran in cells induced to undergo apoptosis. Immunostaining was then carried out on cells transfected with Mst1 siRNA or control siRNA, in the absence and presence of VP16. HeLa cells transfected with Mst1 siRNA demonstrated cytoplasmic localization of Survivin in apoptotic cells (Figure 3c). This evidently indicates that restoration of the RanGTP gradient reinstates the export of Survivin into the cytoplasm of HeLa cells undergoing apoptosis.

Ubiquitinated Survivin are retained in the nucleus pending proteolysis when nuclear export fails during early apoptosis. To verify that the export deregulated Survivin was post-translationally degraded in the nucleus, $\mathrm{CHX}$ was used to block protein synthesis. As shown in Figure $4 \mathrm{a}$, treatment of $\mathrm{CHX}$ in the absence or presence of VP16 exhibited a steady decline in endogenous Survivin level for both the nuclear and also the cytoplasmic fractions. As the Survivin mRNA transcripts are relatively unaffected by VP16 as previously shown (Figure 1b), this data indicated that existing Survivin was actively being degraded when the protein translation process was inhibited. Treatment of proteosome inhibitor MG132 restored both the VP16-treated or non-treated endogenous Survivin protein level over time, particularly in the nuclear fractions (Figure 4b). Western blot illustrated that while Survivin level is stabilized in the cytoplasmic fraction, there is a marked increase of Survivin level in the nuclear fraction over time. More importantly,
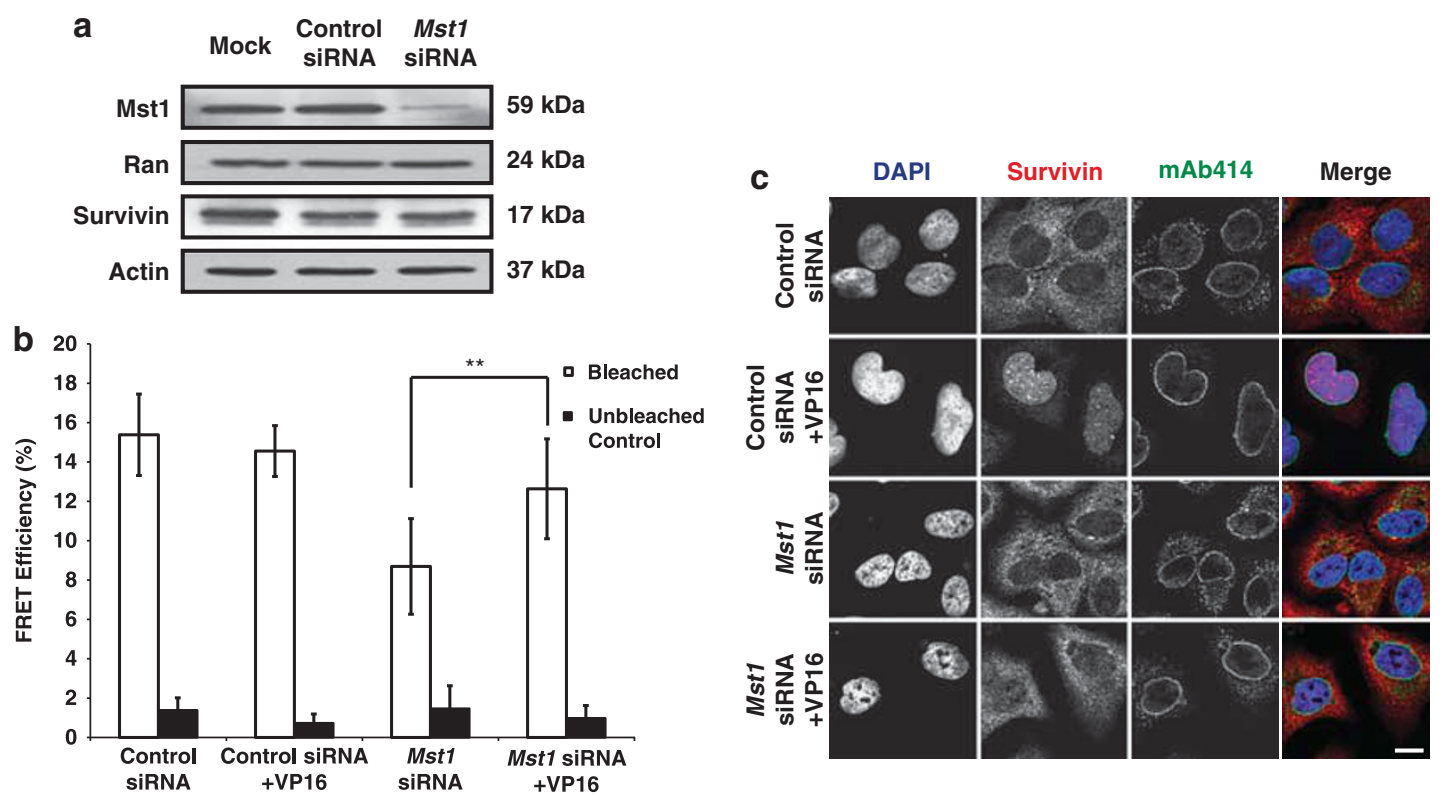

Figure 3 Restoration of RanGTP gradient reinstates Survivin export. (a) Immunoblotting against Mst1, Survivin, Ran and Actin was performed on lysates collected from mock, Control siRNA or Mst1 siRNA-transfected HeLa cells. (b) Rango FRET analysis was performed on Mst1 siRNA or Control siRNA-transfected HeLa cells, with or without VP16 treatment. Quantified data presented as mean \pm S.D. (error bar) of three independent experiments $(n=120) .{ }^{* *} P<0.001$ (Student's $t$-test) (c) HeLa cells transfected with Mst1 siRNA or Control siRNA before $24 \mathrm{~h}$ incubation with VP16. The cells were fixed and stained with anti-Survivin and mAb414. Bar, $10 \mu \mathrm{m}$. Images acquired with auto-optimal exposure mode 


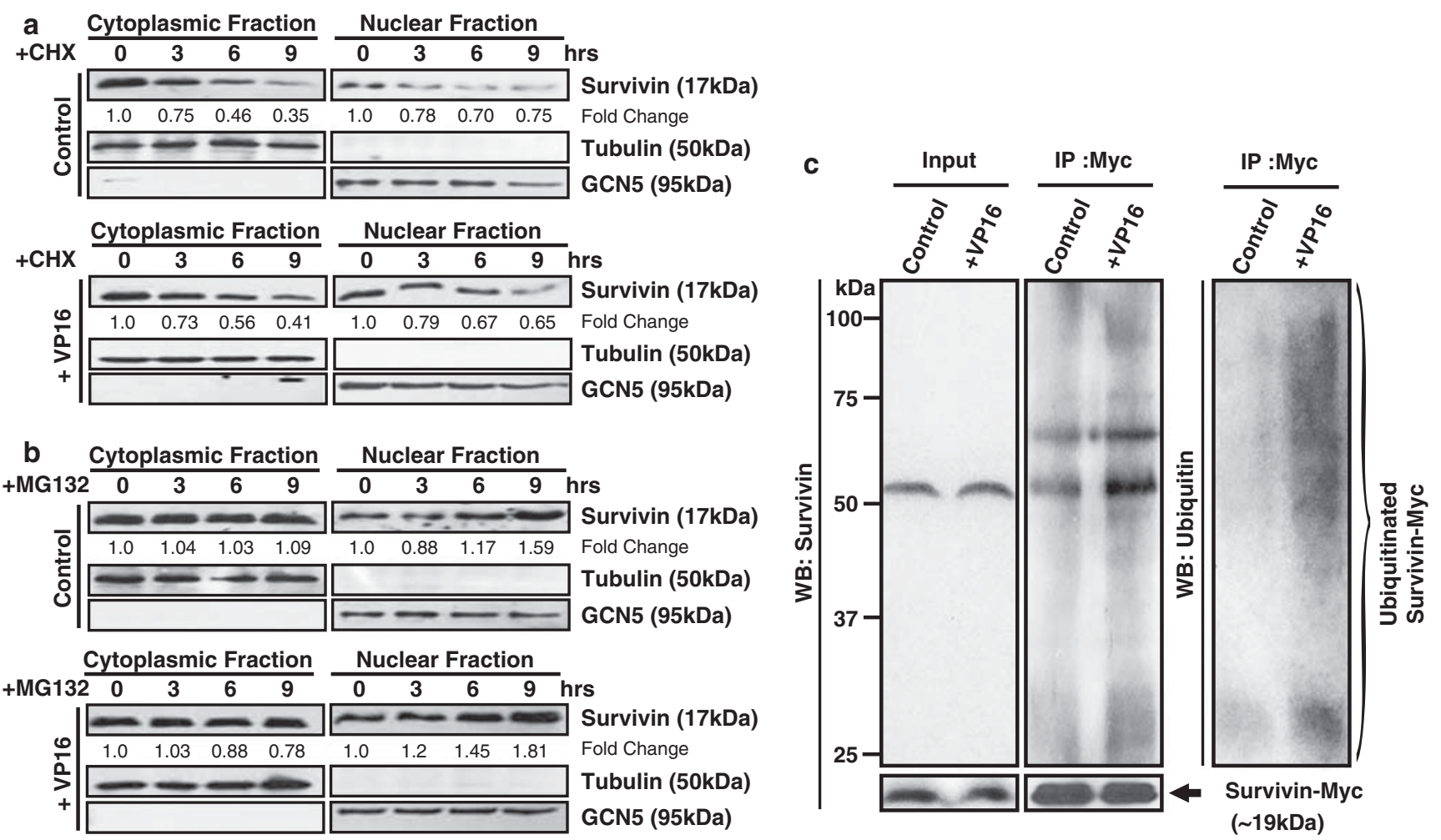

Figure 4 Ubiquitinated Survivin is sequestered and degraded in the nucleus upon apoptosis induction. (a) Nuclearcytoplasmic fractions were collected from $\mathrm{CHX}$-treated cells at the indicated time points. The experiment was repeated on HeLa cells incubated with CHX and VP16 simultaneously. The samples were immunoblotted against Survivin, tubulin and GCN5. (b) Experiment was repeated as in (a) with the exception that CHX is replaced by MG132. (c) Survivin-WT-Myc was overexpressed and immunoprecipitated with anti-Myc antibody from HeLa cells incubated with or without VP16 for $24 \mathrm{~h}$. The immunoprecipitated materials were immunoblotted against Survivin and ubiquitin

exposure to MG132 demonstrated a greater restoration of nuclear Survivin in samples treated with VP16. This suggest that apoptosis accelerates the depletion of prosurvival factor Survivin in the nucleus.

The collapse in RanGTP gradient and hence the failed active transport system during the progression of apoptosis could not fully explain the redistribution of Survivin into the nucleus. Even as the active transport processes ceases, Survivin $(16.5 \mathrm{kDa})$ can readily diffuse across the nuclear membrane until equilibrium is reached between the cytoplasm and the nucleus. However, Survivin is predominantly compartmentalized within the nucleus during apoptosis (Figure 2a and Supplementary Figure S2a). This indicates that Survivin is being sequestered in the nucleus after the export system shuts down.

Upon further inspection, the Survivin stability assay suggested that ubiquitinated Survivin in the nucleus could lead to possible increase in sizes due to multiple conjugations of ubiquitin molecules. NPCs function as selectivity filters that allow molecules $<40 \mathrm{kDa}$ to traverse across the nuclear envelope. We reasoned that after the nuclear transport machinery fails during apoptosis, ubiquitinated Survivin molecules in the nucleus are prevented from diffusing out due to their increased effective molecular weight $>40 \mathrm{kDa}$. Western blot showed that apoptotic samples co-immunoprecipitated greater abundance of ubiquitinated Survivin proteins at various molecular weights above $40 \mathrm{kDa}$ (Figure $4 \mathrm{c}$ ).
Thus, apoptotic relocation of Survivin into the nucleus resulted in multiple ubiquitination of Survivin that increases its molecular weight up to $\sim 100 \mathrm{kDa}$, thereby preventing diffusion across the NPC after nuclear export is impeded.

\section{Survivin cannot prevent apoptosis when compartmen-} talized in the nucleus. During the early stages of apoptosis, Survivin relocates from the cytoplasm into the nucleus due the collapse of RanGTP gradient, whereas both the ProCaspase-3 and activated cleaved Caspase-3 remains in the cytoplasm (Figure $5 \mathrm{a}$ and b). We reasoned that the apoptotic accumulation of Survivin in the nucleus prevents it from interacting with its target proteins in the cytoplasm. As such, the cytoprotectivity of Survivin is compromised even before it is degraded. To study the impact of nuclear Survivin accumulation on apoptosis, we performed the Caspase-3 activity assay using the cleavage of Caspase-3 substrate (Ac-DEVD-AMC) as fluorogenic reporter. Instead of using total lysates to probe for Caspase-3 activity, the cytoplasmic fractions were used in line with prevalence of Caspase-3 in the cytoplasm after apoptosis induction consistent with various reports that demonstrated that Caspase-3 only enters the nucleus during late apoptosis when the nuclear envelope breaks down. As Survivin redistributes into the nucleus during apoptosis, parallel experiments were set up involving a portion of the nuclear fraction being added back to the corresponding cytoplasmic fraction before Caspase-3 
a

\begin{tabular}{|c|c|c|c|c|}
\hline & $\begin{array}{lll}0 & 12 & 24\end{array}$ & $\begin{array}{ll}0 & 12\end{array}$ & 24 & \\
\hline Survivin & 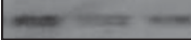 & -5 & - & (16.5 kDa) \\
\hline Tubulin & $m=$ & & & (50 kDa) \\
\hline GCN5 & & +2 & & (95 kDa) \\
\hline & Cytoplasmic & Nucle & & \\
\hline
\end{tabular}

b

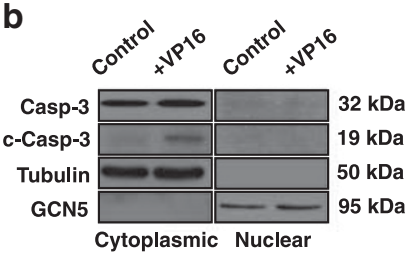

C SiRNA
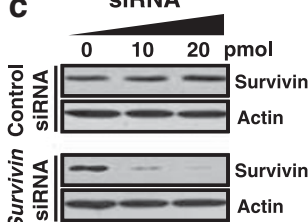

d
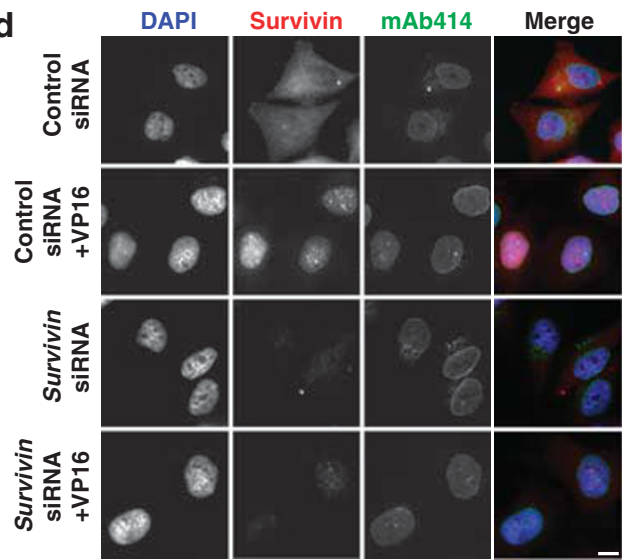

e
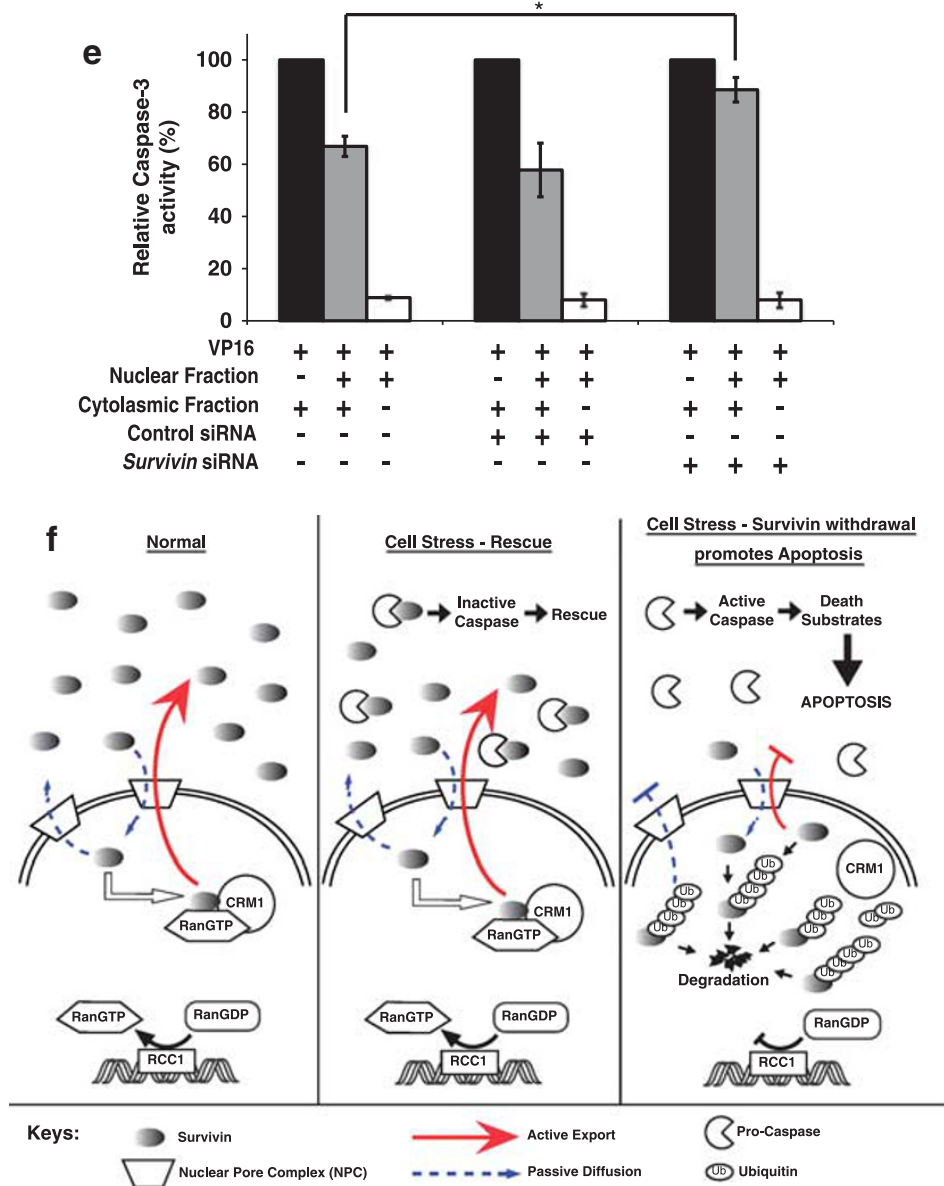

Figure 5 Apoptotic nuclear compartmentalized Survivin has abolished cytoprotectivity. (a) Nuclearcytoplasmic fractions collected from VP16-treated cells at the indicated time points. The samples were immunoblotted against Survivin, tubulin and GCN5. (b) Procaspase-3 and cleaved Caspase-3 are predominantly cytoplasmic in VP16-treated cells indicating early apoptosis. (c) Survivin depletion from HeLa cells by $24 \mathrm{~h}$ Survivin RNAi transfection and immunoblotted against Survivin and Actin. (d) HeLa cells were transfected with Survivin siRNA or Control siRNA before incubation with VP16 for $24 \mathrm{~h}$. Cells were fixed and stained with anti-Survivin and mAb414. Bar, 10 $\mu \mathrm{m}$. (e) Caspase-3 activity assay. Each experiment was performed in triplicates in three independent experiments. Data presented as mean \pm S.D. (error bar). ${ }^{*} P<0.001$ (Student's $t$-test). (f) Hypothetical model depicting the role of nuclear export system in regulating the commitment of cells during the onset of apoptosis

activity readout was taken. As shown in Figure $5 \mathrm{e}$, the addition of Survivin-containing nuclear fractions back its corresponding cytoplasmic fractions yielded significantly lower Caspase-3 activity. To verify that the Caspase-3 inhibitory effect was due to the presence of Survivin in the nuclear fraction, the assay was performed on HeLa cells transfected with Survivin siRNA. Treatment of Survivin siRNA achieved $\sim 90 \%$ knockdown efficiency of the Survivin protein level (Figure $5 \mathrm{c}$ and d). The assay revealed that nuclear fractions depleted of Survivin had little effect on VP16-induced Caspase-3 activity (Figure 5e). In other words, the knockdown of Survivin abolishes the suppresive effect of the supplemented nuclear fraction on cytoplasmic Caspase-3 activity, thus certifying that the confinement of Survivin in the nucleus reduces its cytoprotectivity. This data corroborated other reports that had demonstrated a link between cell death and the nuclear enforcement of Survivin by NES-mutation experiments or the expression of nuclear localization signal-Survivin fusion proteins. ${ }^{19,21,26}$ In addition, transfection of HeLa cells with GST-Survivin-green fluorescent protein (GFP) showed that the mutant Survivin is retained in the cytoplasm due to its increased size from passing through the NPC and reduces the percentage of apoptotic cells in the presence of apoptotic stimuli (Supplementary Figure S5). In summary, the antiapoptotic activity of Survivin is abrogated when it is compartmentalized in the nucleus during the progression of apoptosis.

\section{Discussion}

Apoptosis is a process whereby cells have an active role leading to their own demise (cell suicide). The survival factors function by upregulating the expression and/or activity of antiapoptotic regulatory molecules and maintain the dormancy of apoptotic executors. Upsetting this balance will trigger the cells to initiate the execution of apoptosis. Hence, it is tempting to speculate on the role of the cell, particularly pertaining to the fate of existing prosurvival factors and the molecular events that leads to its subsequent cell death. In this study, we have reported a novel regulatory pathway mediating the active removal of prevailing prosurvival protein 
Survivin during the onset of apoptosis by the impairment of nuclear export machinery.

Survivin is an important bifunctional protein integral as a component of the chromosome passenger complex and also participates in the negative regulation of apoptotic events. We have discovered that the prosurvival protein Survivin is actively being removed by the ubiquitin-proteasome proteolytic pathway upon the exposure to apoptotic stimuli. Moreover, Survivin redistributes from the cytoplasm to the nucleus during the progression of apoptosis. mAb414 staining indicates the preservation of the nuclear envelope integrity throughout the duration of the experiment, thus implying that the function of the nuclear membrane as a selective physical barrier is not compromised.

For a cell under genotoxic stress, it is conceivable that the prosurvival factors will proceed and attempt to rescue the cell from dying. Then again, the cell will probably never die if the prosurvival factor continues to exist and function. Our data demonstrated that the relocation of endogenous Survivin into the nucleus of HeLa cells is a widespread phenomenon during the initial phases of apoptosis. The progressive accumulation of endogenous Survivin in the nucleus was found to be associated to the breakdown of the RanGTP gradient upon apoptosis induction. In addition, this apoptotic nuclear relocalization of Survivin is a recurring phenomenon as observed even in the H1299 cells (Supplementary Figure S6). As a small protein, Survivin is able to shuttle between nuclear and cytoplasmic compartments by passive diffusion under normal circumstances. Nonetheless, the presence of an active NES directs continuous export of Survivin into the cytoplasm by the interaction with its export receptor CRM1. Although most NES by themselves have low affinity for the binding of $\mathrm{CRM} 1{ }^{27}$ the involvement of RanGTP in the formation of the trimeric export complex is necessary for efficient cargo export. Further investigations revealed the redistribution of endogenous Survivin in the nucleus is a consequence of deregulation along the RanGTP-CRM1 axis. The interaction between Survivin and CRM1 was found to be significantly weaker during early apoptosis. This precisely fits the model of cooperative binding between CRM1, RanGTP and the NES-cargo as the basis of the functional trimeric export complex assembly. ${ }^{28,29}$ The decreased nuclear RanGTP level interferes the trimeric RanGTP-CRM1Survivin complex formation and thus leads to diminished export. Conversely, the restoration of RanGTP gradient by Mst1 knockdown reinstated the cytoplasmic localization of endogenous Survivin in apoptotic cells. Hence, we resolved the mechanism behind the failed nuclear export system during the initial phases of apoptosis.

In addition, failure to actively and rapidly transport Survivin back into the cytoplasm helps to justify its decreasing protein level in the course of apoptosis. The presence of Survivin in the nucleus promotes its clearance by the ubiquitinproteasome proteolytic pathway. This corroborates previous findings, which collectively reported the reduced stability of Survivin in the nucleus as a result of proteasomal degradation. ${ }^{19,20}$ More importantly, this implies that the cessation of nuclear export could be the regulatory mechanism for the intentional removal of prosurvival factor Survivin for cells to commit to apoptosis. The conjugation of ubiquitin to Survivin also partially explains how Survivin is retained in the nucleus even after the nuclear export processes are impeded. The small molecular weight of Survivin should theoretically permit passive diffusion back into the cytoplasm even as the RanGTP gradient collapsed. We showed that apoptotic relocation of Survivin into the nucleus resulted in multiple ubiquitination of Survivin that effectively increases its molecular weight up to $\sim 100 \mathrm{kDa}$, thereby prevents diffusion across the NPC.

Survivin has been demonstrated by numerous studies to directly and/or indirectly (by X-linked Inhibitor of apoptosis protein or hepatitis $B$ virus $X$-interacting protein) interact and thus regulate the activities of caspases. ${ }^{1-4,30}$ During interphase, under normal circumstances, Survivin localizes predominantly in the cytoplasm. Therefore, it can interact and inhibit caspases that might be activated when the cell is under stress. However, this does not mean that caspase activity is only confined to the cytoplasm. Although the effector Caspase-3 is found in apoptotic nuclei, this observation is commonly made during the later stages of apoptosis, usually after the integrity of the nuclear membrane is compromised. In recent years, a flurry of studies has associated the nuclear Survivin to the sensitization of viable cells to apoptotic stimuli. ${ }^{9,19,21}$ Here, we demonstrated that endogenous Survivin had limited cytoprotectivity in the nucleus as it was prevented from reaching and interacting with its protein targets (such as the caspases) in the cytoplasm during apoptosis. As described in the results section (Figure 5e), the addition of Survivin-containing nuclear fractions back its corresponding cytoplasmic fractions yielded significantly lower Caspase-3 activity. It appears that the antiapoptotic activity of Survivin is abrogated when it is compartmentalized in the nucleus during the progression of apoptosis.

In conclusion, we have shown here that the nuclear export machinery is impaired. More importantly, our data indicate an unprecedented nuclearcytoplasmic transport associated mechanism to regulate the removal of existing prosurvival factor for cell death to occur (Figure 5f). The abolished cytoprotectivity of Survivin couple to its degradation in the nucleus ensures active withdrawal of positive signals during the early stages of apoptosis. This regulatory mechanism serves as a physiological decision switch, in which the survival balance is consequently tilted and the unrestricted, widespread activation of proapoptotic effector molecules will irreversibly contributes toward apoptosis.

\section{Materials and Methods}

Cell culture and transfection. HeLa cells were obtained from American Type Culture Collection and grown in Dulbecco's modified Eagle's medium (Gibco, Invitrogen, Carlsbad, CA, USA) containing $10 \%$ fetal bovine serum (Hylcone, Themo Scientific, Logan, UT, USA) and 1\% Penicillin/Streptomycin (Gibco, Invitrogen) at $37^{\circ} \mathrm{C}$ in a humidified incubator with $5 \%$ carbon dioxide. Transfections of Rango or Flag-Survivin into HeLa cells were carried out using Lipofectamine 2000, according to manufacturer's instructions (Invitrogen).

Immunofluorescence microscopy. Cells seeded onto $22 \mathrm{~mm}$ coverslips were fixed with $4 \%$ paraformaldehyde in phosphate-buffered saline (PBS) for $10 \mathrm{~min}$ and permeabilized with $0.5 \%$ Triton X-100 (USB, Cleveland, OH, USA) in PBS for $5 \mathrm{~min}$. After washing three times with PBS, cells were blocked in $4 \% \mathrm{BSA}$ (Sigma-Aldrich, St. Louis, MO, USA) for $1 \mathrm{~h}$ at room temperature (RT) and then incubated with primary antibody diluted in TBST ( $50 \mathrm{mM}$ Tris, $\mathrm{pH} 7.6,150 \mathrm{mM} \mathrm{NaCl}$, $0.1 \%$ Tween-20) plus $4 \%$ BSA. Following washes with TBST, the cells were 
incubated with appropriate secondary antibodies and incubated at $\mathrm{RT}$ for $1 \mathrm{~h}$ in the dark. Cells were then mounted onto glass slides using ProLong gold antifade reagent containing 4',6-Diamidino-2-phenylindole (Invitrogen). Images were acquired and analyzed using an Axiovert $200 \mathrm{M}$ inverted microscope (Carl Zeiss, Standort Gottingen, Vertrieb, Germany) and Axiovision 4.6 software. Unless specified, all images are acquired with fixed exposures.

Reagents. For VP16 treatment, $10 \mathrm{mg} / \mathrm{ml}$ VP16 (Sigma-Aldrich) in dimethyl sulfoxide (DMSO) stock was diluted in medium to a final concentration of $20 \mu \mathrm{g} / \mathrm{ml}$. MG132 and CHX (both from Sigma-Aldrich) were diluted with buffers to a final working concentration of $2 \mu \mathrm{M}$ and $20 \mu \mathrm{g} / \mathrm{ml}$, respectively. For LMB (Sigma-Aldrich) treatment, the stock solution of $5 \mu \mathrm{g} / \mathrm{ml}$ in $100 \%$ ethanol was diluted with buffers to a final working concentration of $5 \mathrm{ng} / \mathrm{ml}$.

Antibodies. The antibodies used were anti-Survivin rabbit polyclonal Ab (R\&D Systems, Minneapolis, MN, USA); anti-actin goat polyclonal Ab (Santa Cruz Biotechnology, Santa Cruz, CA, USA); mAb414 mouse monoclonal Ab (Covance Research Product, Princeton, NJ, USA); anti-Ran goat polyclonal Ab (Santa Cruz Biotechnology); anti-Caspase3 rabbit polyclonal Ab (Cell Signaling Technology, Beverly, MA, USA); anti-FLAG M2 mouse monoclonal Ab (Sigma-Aldrich); anti-GCN5 rabbit monoclonal $\mathrm{Ab}$ (Cell Signaling Technology); anti-Myc rabbit monoclonal $\mathrm{Ab}$ (Cell Signaling); anti-cmyc mouse monoclonal Ab (Santa Cruz Biotechnology); anti-Mst1 rabbit polyclonal Ab (Cell Signaling Technology); anti-Ran goat polyclonal $\mathrm{Ab}$ (Santa Cruz Biotechnology); anti-CRM1 mouse monoclonal Ab (BD Biosciences, San Jose, CA, USA); anti- $\alpha$-tubulin mouse monoclonal (Sigma-Aldrich); anti-cleaved DFF45 (Asp224) rabbit polyclonal (Cell Signaling Technology); anti-cleaved PARP (Asp214) rabbit polyclonal (Cell Signaling Technology); Alex Fluor 488 goat anti-rabbit IgG (Invitrogen); Alex Fluor 594 goat anti-rabbit IgG (Invitrogen); and Alex Fluor 488 goat anti-mouse lgG (Invitrogen); horseradish peroxidase-conjugated anti-mouse lgG, anti-rabbit $\lg G$ and anti-goat $\lg G$ (Invitrogen).

Molecular cloning. To create the mammalian expression plasmids, full-length Survivin was amplified (forward primer $5^{\prime}$-TAGGGCGGATCCAGATGGGTGCCCC GACGTTGC- 3 ' and reverse primer $5^{\prime}$-TAGGGCGAATTCTCAATCCATGGCAGCC AGCTG-3') using HeLa CDNA library as template and pfu polymerase (Invitrogen). The cDNA library was generated by reverse transcription-PCR from HeLa total RNA extracts. Survivin amplicons were then subcloned into the pcDNA3-FLAG vector using BamH1 and EcoR1 sites or subcloned into pEF6/myc-His $C$ vector using BamH1 and EcoRV restriction sites. GST-Survivin-GFP contruct was generated by subcloning GST-Survivin into pEGFP-N3 vector using HindIII and EcoR1 sites.

Time-Lapse imaging. Cells were seeded onto $60 \mathrm{~mm}$ dishes and transfected with empty vector or GST-Survivin-GFP for 2 days. The transfected cells were then treated with or without VP16 and placed on a heat-controlled stage of a Zeiss Axiovert $200 \mathrm{M}$ microscope. The temperature was maintained at $37^{\circ} \mathrm{C}$ and $\mathrm{CO}_{2}$ levels were maintained at $5 \%$ using a CTI 3700 controller (Carl Zeiss). Phase contrast and fluorescent images were recorded (AxioCam camera and Axiovision 4.6 software, Carl Zeiss) using a $20 \times$ objective.

FRET. Lab-Tek Chambered cover glasses (Nunc, Rochester, NY, USA) were used to seed the cells. FRET was then performed on a Zeiss LSM 510 Meta confocal microscope equipped with Incubator S (Pecon, Erbach, Germany). During the experiment, the temperature and $\mathrm{CO}_{2}$ level was maintained at $37^{\circ} \mathrm{C}$ and $5 \%$, respectively, by placing the seeded cells in Incubator $\mathrm{S}$ on the microscope stage. Acceptor Photobleaching method was used for FRET analysis of Rango biosensor. ${ }^{31}$ Rango consists of a cyan fluorescence protein (CFP) and a yellow fluorescence protein (YFP) domain flanking an importin- $\beta$ binding domain. In brief, the biosensor will have FRET when RanGTP is present. Five consecutive images were obtained at $1 \%$ of the laser intensity in CFP and YFP channels before and after YFP photobleaching by repeatedly scanning the designated area at $75 \%$ laser intensity. The background CFP fluctuations during FRET were determined from the unbleached control cells present in the same field. FRET efficiency was calculated as $E_{\mathrm{F}} \%=\left(I_{6}-I_{5}\right) \times 100 / I_{6}$, where $I_{5}$ and $I_{6}$ represent the CFP fluorescence intensity of the fifth and sixth images immediately before and after YFP photobleaching. At least 15 cells were analyzed for each experimental set. Results were presented as mean percentage FRET efficiencies \pm S.D.

Preparation of cell extracts, immunoblotting and immunoprecipitation. For the collection of whole cell lysates, HeLa cells were washed with PBS and lysed by M-PER (mammalian protein extraction reagent; Thermo Scientific-Pierce, Rockford, IL, USA) containing 10\% Complete EDTA-free Protease Inhibitor Cocktail (Roche, Basel, Schweiz, Switzerland) and $0.1 \%$ phosphatase cocktail inhibitors 1 and 2 (Sigma-Aldrich). Nuclear cytoplasmic fractions were prepared using NE-PER (nuclear and cytoplasmic extraction) (Thermo Scientific) according to manufacturer's protocol. $\times 2$ sample buffer $(62.5 \mathrm{mM}$ Tris- $\mathrm{HCl}, \mathrm{pH} 6.8,2 \%$ SDS, 25\% glycerol, 5\% 2-mercaptoethanol, 0.01\% bromophenol blue) was added to whole cell lysates, nuclear lysates and cytoplasmic lysates before boiling the mixture for $10 \mathrm{~min}$. Equal amounts of protein were resolved on SDS-polyacrylamide gel (PAGE) and transferred onto nitrocellulose membranes (Bio-Rad Laboratories, Hercules, CA, USA). Membranes were blocked with $10 \%$ skim milk in TBST for $2 \mathrm{~h}$ at RT and probed with specific primary antibodies diluted in blocking solution at $4^{\circ} \mathrm{C}$ overnight. Membranes were washed thrice with TBST before incubating with horseradish peroxidase-conjugated secondary $\lg G$ antibodies (Invitrogen). After washing with TBST, immunoreactive proteins were detected by enhanced chemiluminescence (GE Healthcare-BioSciences, Piscataway, NJ, USA). For immunoprecipitation, HeLa cells stably transfected with Flag-Survivin or SurvivinWt-Myc and HA-Ub were incubated with or without VP16 for $24 \mathrm{~h}$. These cells were then lysed with M-PER and the cell lysates were incubated with the relevant antibody for $1 \mathrm{~h}$ at room temperature. Rec-Protein-G-Sepharose $4 \mathrm{~B}$ (Invitrogen) were added to each experimental set. The resultant mixtures were further incubated for $1 \mathrm{~h}$ at room temperature before repeated washing with Wash buffer (TBS with $100 \mathrm{mM} \mathrm{NaCl}$ ) to rid unbound proteins. Immunoprecipitated proteins were separated by SDS-PAGE before western blotting analyses.

RNAi-mediated silencing of Mst1 and Survivin. Stealth select RNAi for Mst1 and Survivin (both from Invitrogen) were used to deplete Mst1 and Survivin protein level, respectively, in HeLa cells. Mst1 silencing was carried out using $10 \mathrm{nM}$ of the oligo (AAUGAUAUCAGAUACAGAACCAGCC). Survivin depletion on the other hand was performed using $10 \mathrm{nM}$ of the oligo (HSS141245). RNAi negative control duplexes (Invitrogen) served as negative control in each experimental set. The indicated RNAi were introduced into the cells by transfection using Lipofectamine RNAiMAX (Invitrogen). The transfected cells were then harvested $24 \mathrm{~h}$ later and resuspended in $\times 2$ SDS sample buffer and boiled. Depletion was assessed by immunoblotting with the relevant antibodies.

Semiquantitative reverse transcription-PCR. Total RNA was extracted from HeLa cells using the RNeasy kit (QIAGEN Inc., Valencia, CA, USA) according to the manufacturer protocol. Total RNA from non-treated and treated samples according to the time indicated were used for the preparation of first-strand CDNA by reverse transcription using the MMLV-based reverse transcriptase (Stratagene, La Jolla, CA, USA). Human Survivin mRNA was amplified using the forward primer $5^{\prime}$-TAGGGCGAATTCATGGGTGCCCCGGAC GTTGC-3' and the reverse primer $5^{\prime}$-TAGGGCGGATCCTCAATCCATGGCAGC CAGCTG-3' (corresponding to full-length Survivin sequence). GAPDH was also amplified as an internal control. The PCR cycling conditions were set to 25 rounds to prevent amplification beyond the exponential phase. Reaction products were analyzed on $1.0 \%$ agarose gels containing ethidium bromide.

Survivin stability assay. MG132 (Sigma-Aldrich) was used to accumulate a pool of endogenous Survivin proteins from HeLa cells treated with or without VP16. The cells were then washed three times with PBS to lift the proteasome inhibitory effects of MG132. After MG132 washoff, the productions of new Survivin proteins were terminated by the addition of $\mathrm{CHX}$ (Sigma-Aldrich). SDS-PAGE and western blot were performed against Survivin for analysis and quantification at each indicated time points after MG132 release.

Caspase-3 activity assay and analyses. Nuclearcytoplasmic fractions were prepared as described above from HeLa cells transfected Control RNAi, Survivin RNAi or mock transfected, with or without VP16 treatment. Lysates were snap-frozen and stored at $-80^{\circ} \mathrm{C}$. Ninety-six-well microtiter plates (Nunc) were used for this assay together with the caspase-3 substrate, fluorogenic tetrapeptide Ac-DEVD-AMC (Sigma-Aldrich). A quantity of $5 \mathrm{ng}$ of the substrate was incubated at $37^{\circ} \mathrm{C}$ for $3 \mathrm{~h}$ in $200 \mu \mathrm{l}$ of reaction buffer ( $20 \mathrm{mM}$ HEPES, $\mathrm{pH} 7.4$, with $2 \mathrm{mM}$ EDTA, $0.1 \%$ CHAPS and $5 \mathrm{mM} \mathrm{DTT}$ ) and $10 \mu \mathrm{l}$ of cytoplasmic extracts with or without $5 \mu \mathrm{l}$ of the corresponding nuclear extracts. Spectrofluorometer measured fluorescence produced by substrate cleavage with excitation and emission wavelength set at 355 and $460 \mathrm{~nm}$, respectively. For each experimental set-up, results were expressed as the mean relative percentage of caspase- 3 activity between samples 
containing only the cytoplasmic extracts and samples containing both cytoplasmic and nuclear extracts. For better and more accurate comparison, the read-outs obtained from cytoplasmic extract only samples were set as the absolute value $(100 \%)$, such that the read-outs from the corresponding samples containing both the cytoplasmic and nuclear extracts can be normalized accordingly.

\section{Conflict of interest}

The authors declare no conflict of interest.

Acknowledgements. We thank $\operatorname{Dr} Y$ Zheng for Survivin-Myc construct. This work was supported by A-STAR Biomedical Research Council (BMRC) Grant, Singapore $(08 / 1 / 22 / 19 / 568)$.

1. Kobayashi K, Hatano M, Otaki M, Ogasawara T, Tokuhisa T. Expression of a murine homologue of the inhibitor of apoptosis protein is related to cell proliferation. Proc Natl Acad Sci USA 1999; 96: 1457-1462.

2. Marusawa $\mathrm{H}$, Matsuzawa S, Welsh $\mathrm{K}$, Zou H, Armstrong R, Tamm I et al. HBXIP functions as a cofactor of survivin in apoptosis suppression. EMBO J 2003; 22: 2729-2740.

3. Shin S, Sung BJ, Cho YS, Kim HJ, Ha NC, Hwang Jl et al. An anti-apoptotic protein human survivin is a direct inhibitor of caspase-3 and -7. Biochemistry 2001; 40: 1117-1123.

4. Tamm I, Wang Y, Sausville E, Scudiero DA, Vigna N, Oltersdorf T et al. IAP-family protein survivin inhibits caspase activity and apoptosis induced by Fas (CD95), Bax, caspases, and anticancer drugs. Cancer Res 1998; 58: 5315-5320.

5. Wheatley SP, McNeish IA. Survivin: a protein with dual roles in mitosis and apoptosis. Int Rev Cytol 2005; 247: 35-88.

6. Ferrandina G, Legge F, Martinelli E, Ranelletti FO, Zannoni GF, Lauriola L et al. Survivin expression in ovarian cancer and its correlation with clinico-pathological, surgical and apoptosis-related parameters. Br J Cancer 2005; 92: 271-277.

7. Kennedy SM, O'Driscoll L, Purcell R, Fitz-Simons N, McDermott EW, Hill AD et al. Prognostic importance of survivin in breast cancer. Br J Cancer 2003; 88: 1077-1083

8. Okada E, Murai Y, Matsui K, Isizawa S, Cheng C, Masuda M et al. Survivin expression in tumor cell nuclei is predictive of a favorable prognosis in gastric cancer patients. Cancer Lett 2001; 163: 109-116.

9. Temme A, Rodriguez JA, Hendruschk S, Gunes S, Weigle B, Schakel K et al. Nuclea localization of Survivin renders HeLa tumor cells more sensitive to apoptosis by induction of p53 and Bax. Cancer Lett 2007; 250: 177-193.

10. Tonini G, Vincenzi B, Santini D, Scarpa S, Vasaturo T, Malacrino C et al. Nuclea and cytoplasmic expression of survivin in 67 surgically resected pancreatic cancer patients. Br J Cancer 2005; 92: 2225-2232.

11. Mita AC, Mita MM, Nawrocki ST, Giles FJ. Survivin: key regulator of mitosis and apoptosis and novel target for cancer therapeutics. Clin Cancer Res 2008; 14: 5000-5005.

12. Stauber RH, Mann W, Knauer SK. Nuclear and cytoplasmic survivin: molecula mechanism, prognostic, and therapeutic potential. Cancer Res 2007; 67: 5999-6002.

13. Chakravarti A, Zhai GG, Zhang M, Malhotra R, Latham DE, Delaney MA et al. Survivin enhances radiation resistance in primary human glioblastoma cells via caspaseindependent mechanisms. Oncogene 2004; 23: 7494-7506.
14. Lu B, Mu Y, Cao C, Zeng F, Schneider S, Tan J et al. Survivin as a therapeutic target for radiation sensitization in lung cancer. Cancer Res 2004; 64: 2840-2845.

15. Knauer SK, Mann W, Stauber RH. Survivin's dual role: an export's view. Cell Cycle 2007; 6 : 518-521.

16. Rodriguez JA, Span SW, Ferreira CG, Kruyt FA, Giaccone G. CRM1-mediated nuclear export determines the cytoplasmic localization of the antiapoptotic protein Survivin. Exp Cell Res 2002; 275: 44-53.

17. Stauber RH, Rabenhorst U, Rekik A, Engels K, Bier C, Knauer SK. Nucleocytoplasmic shuttling and the biological activity of mouse survivin are regulated by an active nuclear export signal. Traffic 2006; 7: 1461-1472.

18. Knauer SK, Kramer OH, Knosel T, Engels K, Rodel F, Kovacs AF et al. Nuclear export is essential for the tumor-promoting activity of survivin. FASEB J 2007; 21: 207-216.

19. Connell CM, Colnaghi R, Wheatley SP. Nuclear survivin has reduced stability and is not cytoprotective. J Biol Chem 2008; 283: 3289-3296.

20. Zhao J, Tenev T, Martins LM, Downward J, Lemoine NR. The ubiquitin-proteasome pathway regulates survivin degradation in a cell cycle-dependent manner. J Cell Sci 2000; 113 (Part 23): 4363-4371.

21. Colnaghi R, Connell CM, Barrett RM, Wheatley SP. Separating the anti-apoptotic and mitotic roles of survivin. J Biol Chem 2006; 281: 33450-33456.

22. Kihlmark M, Imreh G, Hallberg E. Sequential degradation of proteins from the nuclear envelope during apoptosis. J Cell Sci 2001; 114 (Part 20): 3643-3653.

23. Walther TC, Fornerod M, Pickersgill H, Goldberg M, Allen TD, Mattaj IW. The nucleoporin Nup153 is required for nuclear pore basket formation, nuclear pore complex anchoring and import of a subset of nuclear proteins. EMBO J 2001; 20: 5703-5714.

24. Mosammaparast N, Pemberton LF. Karyopherins: from nuclear-transport mediators to nuclear-function regulators. Trends Cell Biol 2004; 14: 547-556.

25. Wong $\mathrm{CH}$, Chan $\mathrm{H}, \mathrm{Ho} \mathrm{CY}$, Lai SK, Chan KS, Koh CG et al. Apoptotic histone modification inhibits nuclear transport by regulating RCC1. Nat Cell Biol 2009; 11: 36-45.

26. Li F, Yang J, Ramnath N, Javle MM, Tan D. Nuclear or cytoplasmic expression of survivin: what is the significance? Int J Cancer 2005; 114: 509-512.

27. Kutay U, Guttinger S. Leucine-rich nuclear-export signals: born to be weak. Trends Cell Biol 2005; 15: 121-124

28. Hutten S, Kehlenbach RH. CRM1-mediated nuclear export: to the pore and beyond. Trends Cell Biol 2007; 17: 193-201.

29. Petosa C, Schoehn G, Askjaer P, Bauer U, Moulin M, Steuerwald U et al. Architecture of CRM1/Exportin1 suggests how cooperativity is achieved during formation of a nuclear export complex. Mol Cell 2004; 16: 761-775.

30. Dohi T, Okada K, Xia F, Wilford CE, Samuel T, Welsh K et al. An IAP-IAP complex inhibits apoptosis. J Biol Chem 2004; 279: 34087-34090.

31. Li HY, Zheng Y. Phosphorylation of RCC1 in mitosis is essential for producing a high RanGTP concentration on chromosomes and for spindle assembly in mammalian cells. Genes Dev 2004; 18: 512-527.

Cell Death and Disease is an open-access journal published by Nature Publishing Group. This work is licensed under the Creative Commons Attribution-Noncommercial-No Derivative Works 3.0 Unported License. To view a copy of this license, visit http://creativecommons.org/licenses/by-nc-nd/3.0/

\section{Supplementary Information accompanies the paper on Cell Death and Disease website (http://www.nature.com/cddis)}

